\title{
A Qualitative Study of Increased Pediatric Reutilization After a Postdischarge Home Nurse Visit
}

\author{
Sarah W Riddle, MD, IBCLC $1,2 \star$, Susan N Sherman, DPA3 ${ }^{3}$ Margo J Moore, MS, BSN, RN ${ }^{4}$, \\ Allison M Loechtenfeldt, BS ${ }^{1}$, Heather L Tubbs-Cooley, PhD, RN 5 , Jennifer M Gold, BSN, RN ${ }^{4}$, \\ Susan Wade-Murphy, MSN, RN ${ }^{4}$, Andrew F Beck, MD, MPH ${ }^{1,2,6}$, Angela M Statile, MD, MEd ${ }^{1,2}$, Samir S Shah, MD, MSCE ${ }^{1,2}$, \\ Jeffrey M Simmons, MD, MSc $c^{1,2,7}$, Katherine A Auger, MD, MSc ${ }^{1,2,7}$ on behalf of the $\mathrm{H} 2 \mathrm{O}$ study group
}

${ }^{1}$ Division of Hospital Medicine, Cincinnati Children's Hospital Medical Center, Cincinnati, Ohio; ${ }^{2}$ Department of Pediatrics, University of Cincinnati College of Medicine, Cincinnati, Ohio; ${ }^{3}$ SNS Research, Cincinnati, Ohio; ${ }^{4}$ Division of Patient Services, Cincinnati Children's Hospital Medical Center, Cincinnati, Ohio; ${ }^{5}$ College of Nursing, Martha S. Pitzer Center for Women, Children and Youth, Columbus, Ohio; ${ }^{6}$ Division of General and Community Pediatrics, Cincinnati Children's Hospital Medical Center, Cincinnati, Ohio; ${ }^{7}$ James M. Anderson Center for Health System Excellence, Cincinnati Children's Hospital Medical Center, Cincinnati, Ohio.

BACKGROUND: The Hospital to Home Outcomes (H2O) trial was a 2-arm, randomized controlled trial that assessed the effects of a nurse home visit after a pediatric hospital discharge. Children randomized to the intervention had higher 30-day postdischarge reutilization rates compared with those with standard discharge. We sought to understand perspectives on why postdischarge home nurse visits resulted in higher reutilization rates and to elicit suggestions on how to improve future interventions.

METHODS: We sought qualitative input using focus groups and interviews from stakeholder groups: parents, primary care physicians (PCP), hospital medicine physicians, and home care registered nurses (RNs). A multidisciplinary team coded and analyzed transcripts using an inductive, iterative approach.

RESULTS: Thirty-three parents participated in interviews. Three focus groups were completed with PCPs $(n=$ 7), 2 with hospital medicine physicians $(n=12)$, and 2 with RNs $(n=10)$. Major themes in the explanation of increased reutilization included: appropriateness of patient reutilization; impact of red flags/warning sign instructions on family's reutilization decisions; hospital-affiliated RNs "directing traffic" back to hospital; and home visit RNs had a low threshold for escalating care. Major themes for improving design of the intervention included: need for improved postdischarge communication; individualizing home visits-one size does not fit all; and providing context and framing of red flags.

CONCLUSION: Stakeholders questioned whether hospital reutilization was appropriate and whether the intervention unintentionally directed patients back to the hospital. Future interventions could individualize the visit to specific needs or diagnoses, enhance postdischarge communication, and better connect patients and home nurses to primary care. Journal of Hospital Medicine 2020;15:518-525. () 2020 Society of Hospital Medicine eadmission rates are used as metrics for care quality and reimbursement, with penalties applied to hospitals with higher than expected rates ${ }^{1}$ and up to $30 \%$ of pediatric readmissions deemed potentially preventable. ${ }^{2}$ There is a paucity of information on how to prevent pediatric readmissions, ${ }^{3}$ yet pediatric hospitals are tasked with implementing interventions for readmission reduction.

The Hospital to Home Outcomes $(\mathrm{H} 2 \mathrm{O})$ trial was a 2-arm, randomized controlled trial in which patients discharged from hospital medicine and neuroscience services at a single institution were randomized to receive a single home visit from a registered nurse (RN) within 96 hours of discharge. ${ }^{4}$ RNs completed a structured

*Corresponding Author: Sarah W Riddle, MD, IBCLC; Email: sarah.riddle@ cchmc.org; Telephone: 513-636-1003.

Published online first March 18, 2020

Find additional supporting information in the online version of this article.

Received: July 18, 2019; Revised: December 5, 2019;

Accepted: December 7, 2019

๑) 2020 Society of Hospital Medicine DOI 10.12788/jhm.3370 nurse visit designed specifically for the trial. Lists of "red flags" or warning signs associated with common diagnoses were provided to assist RNs in standardizing education about when to seek additional care. The hypothesis was that the postdischarge visits would result in lower reutilization rates (unplanned readmissions, emergency department [ED] visits, and urgent care visits). ${ }^{5}$

Unexpectedly, children randomized to receive the postdischarge nurse visit had higher rates of 30-day unplanned healthcare reutilization, with children randomly assigned to the intervention demonstrating higher odds of 30-day healthcare use (OR 1.33; 95\% $\mathrm{Cl} 1.003-1.76) .{ }^{4}$ We sought to understand perspectives on these unanticipated findings by obtaining input from relevant stakeholders. There were 2 goals for the qualitative analysis: first, to understand possible explanations of the increased reutilization finding; second, to elicit suggestions for improving the nurse visit intervention.

\section{METHODS}

We selected an in-depth qualitative approach, using interviews and focus groups to explore underlying explanations 
for the increase in 30-day unplanned healthcare reutilization among those randomized to receive the postdischarge nurse visit during the $\mathrm{H} 2 \mathrm{O}$ trial. ${ }^{4}$ Input was sought from 4 stakeholder groups-parents, primary care physicians (PCPs), hospital medicine physicians, and home care RNs-in an effort to triangulate data sources and elicit rich and diverse opinions. Approval was obtained from the Institutional Review Board prior to conducting the study.

\section{Recruitment}

\section{Parents}

Because we conducted interviews approximately 1 year after the trial's conclusion, we purposefully selected families who were enrolled in the latter portion of the $\mathrm{H} 2 \mathrm{O}$ trial in order to enhance recall. Beginning with the last families in the study, we sequentially contacted families in reverse order. We contacted 10 families in each of 4 categories (intervention/reutilization, intervention/no reutilization, control/reutilization, control/no reutilization). A total of 3 attempts were made by telephone to contact each family. Participants received a grocery store gift card for participating in the study.

\section{Primary Care Physicians}

We conducted focus groups with a purposive sample of physicians recruited from 2 community practices and 1 hospitalowned practice.

\section{Hospital Medicine Physicians}

We conducted focus groups with a purposive sample of physicians from our Division of Hospital Medicine. There was a varying level of knowledge of the original trial; however, none of the participants was a collaborator in the trial.

\section{Home Care RNs}

We conducted focus groups with a subset of RNs who were involved with trial visits. All RNs were members of the pediatric home care division associated with the hospital with specific training in caring for patients at home.

\section{Data Collection}

The study team designed question guides for each stakeholder group (Appendix 1). While questions were tailored for specific stakeholders, all guides included the following topics: benefits and challenges of nurse visits, suggestions for improving the intervention in future trials, and reactions to the trial results (once presented to participants). Only the results of the intention-to-treat (ITT) analysis were shared with stakeholders because ITT is considered the gold standard for trial analysis and allows easy understanding of the results.

A single investigator (A.L.) conducted parental interviews by telephone. Focus groups for PCPs, hospital medicine physicians, and RN groups were held at practice locations in private conference rooms and were conducted by trained moderators (S.N.S., A.L., and H.T.C.). Moderators probed responses to the open-ended questions to delve deeply into issues. The question guides were modified in an iterative fashion to include new concepts raised during interviews or focus groups. All interviews and focus groups were recorded and transcribed verbatim with all identifiable information redacted.

\section{Data Analysis}

During multiple cycles of inductive thematic analysis, ${ }^{6}$ we examined, discussed, interpreted, and organized responses to the open-ended questions, ${ }^{6,7}$ analyzing each stakeholder group separately. First, transcripts were shared with and reviewed by the entire multidisciplinary team (12 members), which included hospital medicine physicians, PCPs, home care nursing leaders, a nurse scientist, a parent representative, research coordinators, and a qualitative research methodologist. Second, team members convened to discuss overall concepts and ideas and created the preliminary coding frameworks. Third, a smaller subgroup (research coordinator [A.L.], hospital medicine physician [S.R.], parent representative [M.M.], and qualitative research methodologist [S.N.S.]), refined the unique coding framework for each stakeholder group and then independently applied codes to participant comments. This subgroup met regularly to reach consensus about the assigned codes and to further refine the codebooks. The codes were organized into major and minor themes based on recurring patterns in the data and the salience or emphasis given by participants. The subgroup's work was reviewed and discussed on an ongoing basis by the entire multidisciplinary team. Triangulation of the data was achieved in multiple ways. The preliminary results were shared in several forums, and feedback was solicited and incorporated. Two of 4 members of the subgroup analytic team were not part of the trial planning or data collection, providing a potentially broader perspective. All coding decisions were maintained in an electronic database, and an audit trail was created to document codebook revisions.

\section{RESULTS}

A total of 33 parents participated in the interviews (intervention/readmit [8], intervention/no readmit [8], control/readmit [8], and control/no readmit [9]). Although we selected families from all 4 categories, we were not able to explore qualitative differences between these groups because of the relatively low numbers of participants. Parent data were very limited because interviews were brief and "control" parents had not received the intervention. Three focus groups were held with PCPs (7 participants in total), 2 focus groups were held with hospital medicine physicians (12 participants), and 2 focus groups were held with RNs (10 participants).

\section{Goal 1: Explanation of Reutilization Rates}

During interviews and focus groups, the results of the $\mathrm{H} 2 \mathrm{O}$ trial were discussed, and stakeholders were asked to comment on potential explanations of the findings. Four major themes and 5 minor themes emerged from analysis of the transcripts (summarized in Table 1).

\section{Theme 1: Appropriateness of Patient Reutilization}

Hospital medicine physicians and home care RNs questioned 
TABLE 1. Summary of Major and Minor Themes by Stakeholder Type

\begin{tabular}{|c|c|c|c|c|}
\hline & Parents & Hospital Medicine Physicians & Primary Care Physicians & $\begin{array}{l}\text { Home Healthcare } \\
\text { Nurses }\end{array}$ \\
\hline \multicolumn{5}{|l|}{ Goal 1: Explanation of Reutilization Rates } \\
\hline \multicolumn{5}{|l|}{ Major Themes } \\
\hline Appropriateness of patient reutilization & $\checkmark$ & $\checkmark$ & & $\checkmark$ \\
\hline Impact of red flags on family's reutilization decisions & $\checkmark$ & $\checkmark$ & & $\checkmark$ \\
\hline \multicolumn{5}{|l|}{ Minor Themes } \\
\hline Families receiving visit might perceive that their child was sicker & & & $\checkmark$ & $\checkmark$ \\
\hline Patients in the control group did not reutilize enough & & & $\checkmark$ & $\checkmark$ \\
\hline Receiving more education drives reutilization & & & & $\checkmark$ \\
\hline Provider access issues & & & & $\checkmark$ \\
\hline \multicolumn{5}{|l|}{ Goal 2: Suggestions for Improving the Intervention } \\
\hline \multicolumn{5}{|l|}{ Major Themes } \\
\hline Need for improved postdischarge communication & $\checkmark$ & $\checkmark$ & $\checkmark$ & $\checkmark$ \\
\hline Individualizing home visits—one size does not fit all & $\checkmark$ & $\checkmark$ & $\checkmark$ & $\checkmark$ \\
\hline Providing context and framing of red flags & & & $\checkmark$ & $\checkmark$ \\
\hline \multicolumn{5}{|l|}{ Minor Themes } \\
\hline Streamlining discharge & & & & $\checkmark$ \\
\hline Improving the definition of the scope and goal of intervention & & $\checkmark$ & & \\
\hline Extending inpatient team expertise postdischarge & & $\checkmark$ & & \\
\hline
\end{tabular}

Abbreviation: RN, registered nurses

whether the reutilization events were clinically indicated. RNs wondered whether children who reutilized the ED were also readmitted to the hospital; many perceived that if the child was ill enough to be readmitted, then the ED revisit was warranted (Table 2). Parents commented on parental decision-making and changes in clinical status of the child leading to reutilization (Table 2).

\section{Theme 2: Impact of Red Flags/Warning Sign Instructions on Family's Reutilization Decisions}

Hospital medicine physicians and RNs discussed the potential concern that the "red flags" lacked sufficient context for appropriate family decision making. They hypothesized that, as a result, parents might have returned to the ED rather than accessing other avenues of care such as their primary care office. For example, one participant noted that, if a fever recurred days after it had resolved, then perhaps that would require different action steps than if a child had a persistent fever. RNs also mentioned that the discussion of red flags may have made families "more diligent" (Table 2).

\section{Theme 3: Hospital-Affiliated RNs "Directing Traffic" Back to Hospital}

Both physician groups were concerned that, because the study was conducted by hospital-employed nurses, families might have been more likely to reaccess care at the hospital. Thus, the connection with the hospital was strengthened in the $\mathrm{H} 2 \mathrm{O}$ model, potentially at the expense of the connection with PCPs. Physicians hypothesized that families might "still feel part of the medical system," so families would return to the hospital if there was a problem. PCPs emphasized that there may have been straightforward situations that could have been handled appropriately in the outpatient office (Table 2).

\section{Theme 4: Home Visit RNs Had a Low Threshold for Escalating Care}

Parents and PCPs hypothesized that RNs are more conservative and, therefore, would have had a low threshold to refer back to the hospital if there were concerns in the home. One parent commented: "I guess, nurses are just by trade accustomed to erring on the side of caution and medical intervention instead of letting time take its course.... They're more apt to say it's better off to go to the hospital and have everything be fine" (Table 2).

\section{Minor Themes}

Participants also explained reutilization in ways that coalesced into 5 minor themes: (1) families receiving a visit might perceive that their child was sicker; (2) patients in the control group did not reutilize enough; (3) receiving more education on a child's illness drives reutilization; (4) provider access issues; and (5) variability of RN experience may determine whether escalated 
TABLE 2. Explanation of Reutilization Rates: Major Themes and Illustrative Quotations

Theme
Appropriateness of patient reutilization

"Overconcerned parents, honestly, because ... when any doctor or any nurse or anybody comes to you and, you know, basically, here's your risk, you know, and then you start thinking about them. And you're like, 'Oh, my God, it could be the worst thing possible' ... but, realistically, do you stop thinking about it? No, you don't. ... I mean, as a parent, you're always worried about your kids.... We just know a little bit more information now that we didn't know, which is good to know. ... I mean, once your kid is sick and they've been sick off and on, it starts to wear on you."

HM "Because that would be interesting if like, if it was a failure to connect with the PCP about an issue that maybe wasn't truly emergent but needed to be dealt with at some point that day, if they weren't able to get ahold of the PCP and that was why they re-presented."

"It becomes the piece of what is, what would be meaningful reutilization, and I don't think we have a way to measure that."

"Because a lot of times we get admissions that we don't necessarily think are indicated, but they get admitted because the parents show up in the ED 3 times over 3 days."

RN "Did all of those people who came back to the emergency room need to be back in the emergency room?"

"If they got readmitted, then they obviously needed to be at the emergency room."

Impact of "red flags" on family's reutilization Parent decisions
HM

PN

RN
[Nurses said:] "They should be better by day 5, and then the kids weren't. Or they should, if they have these returning symptoms, you should definitely come back."

"This is a parent, their third or fourth child, they've been through this before, and to see their eyes kind of pop open when somebody mentions something unexpected like, 'You want me to come to the emergency department if there's a fever?' Like, and then you can see the wheels turning like, 'Oh, okay, I mean, I can do that if that's what I'm supposed to do,' without that clarification of, well, there's context to that that's really important."

"Yeah, it makes you wonder if like, if we're too conservative with our red flags [warning signs] if the kids are, you know, with bronchiolitis are still surviving even though they might be living out there with a red flag or two."

"But if the fevers completely go away and are gone for 3 or 4 days, then I want you to start the clock over."

"They [intervention parents] were more diligent because they had the red flags [warning signs]."

"Okay, so maybe we could stress to them a little more about, hey, you know, unless it's a red flag [warning signs], I mean, I feel like we kind of already do that, you don't need to go to the emergency room, unless it's Friday night."

"Maybe I [RN] overalerted families when I went over the red flags. Maybe there's a way that I could phrase things differently" care. Supportive quotations are found in Appendix 2.

We directly asked parents if they would want a nurse home visit in the future after discussing the results of the study. Almost all of the parents in the intervention group and most of the parents in the control group were in favor of receiving a visit, even knowing that patients who had received a visit were more likely to reutilize care.

\section{Goal 2: Suggestions for Improving Intervention Design}

Three major themes and 3 minor themes were related to improving the design of the intervention (Table 1).

\section{Theme 1: Need for Improved Postdischarge Communication}

All stakeholder groups highlighted postdischarge communication as an area that could be improved. Parents were frustrated with regard to attempts to connect with inpatient physicians after discharge. PCPs suggested developing pathways for the $\mathrm{RN}$ to connect with the primary care office as opposed to the hospital. Hospital medicine physicians discussed a lack of consensus regarding patient ownership following discharge and were uncertain about what types of postdischarge symptoms PCPs would be comfortable managing. RNs described specific situations when they had difficulty contacting a physician to escalate care (Table 3 ).

\section{Theme 2: Individualizing Home Visits-One Size Does Not Fit All}

All stakeholder groups also encouraged "individualization" of home visits according to patient and family characteristics, diagnosis, and both timing and severity of illness. PCPs recommended visits only for certain diagnoses. Hospital medicine physicians voiced similar sentiments as the PCPs and added that worrisome family dynamics during a hospitalization, such as a lack of engagement with the medical team, might also warrant a visit. RNs suggested visits for those families with more concerns, for example, those with young children or children recovering from an acute respiratory illness (Table 3 ). 
TABLE 2. Explanation of Reutilization Rates: Major Themes and Illustrative Quotations (continued)

\begin{tabular}{|c|c|c|}
\hline Theme & Stakeholder Type & Illustrative Quotation \\
\hline \multirow[t]{4}{*}{$\begin{array}{l}\text { Hospital-affiliated RNs "directing traffic" back } \\
\text { to hospital }\end{array}$} & $\mathrm{HM}$ & $\begin{array}{l}\text { "The fact that the nurses were a hospital-based versus medical home-based ... might have biased patients going to more } \\
\text { expensive resources like ER versus the primary care doctor." }\end{array}$ \\
\hline & & $\begin{array}{l}\text { "I mean, I think they still feel part of the medical system in that they're still under medical care, and ... like that transition back } \\
\text { to the emergency room doesn't seem as big when you're still under care. ... It may also circumvent the primary care physician, so } \\
\text { their next step in seeking help might be the emergency department instead of the primary care physician because they are liaising } \\
\text { more with us and more attached to the hospital than they are with the primary care doc." }\end{array}$ \\
\hline & & $\begin{array}{l}\text { "There's a concept in the healthcare business world that they call 'Build it and they will come.' And the essential observation } \\
\text { there is that utilization drives even more utilization." }\end{array}$ \\
\hline & PCP & $\begin{array}{l}\text { "Like I wonder if you looked at how involved the PCPs were in directing traffic, or was it because it's a study done by Children's, } \\
\text { now Children's is directing the traffic, and the family, therefore, is returning to Children's versus returning to their PCP." }\end{array}$ \\
\hline
\end{tabular}

"So if this is a bread-and-butter, general pediatric thing, they should be calling the PCP because, once they are really gone, I mean, hospital medicine doctor, we're now siloed except for our group. They're focused on what is admission criteria. And they don't always know how long things take to get better or the twists and turns it might take because they're seeing it just on that side. We're seeing both ends, so we have a good idea about that."

"It's so much about who the relationship is with, right?"

Home visit RNs had a low threshold for Parent

"The only thing that I could think of is maybe based on advice from the nurse that maybe you should return if, you know, your child is exhibiting certain symptoms or ... with me, a lot of times like I won't go to the hospital because I may think that something is not serious enough or, you know, it's not warranted for a trip to the hospital, when in reality it may be."

"The only thing I could really think of is if they maybe pointed something out that was wrong with the kid or that seemed to not be right, and so they were going, they would call when they saw that or call right, you know, take them in because the nurse said, hey, this is not right or this, you might want to get this checked out, or something like that. It may have been based on the nurse's, what the nurse saw."

"I guess, nurses are just by trade accustomed to erring on the side of caution and medical intervention instead of letting time take its course. ... They're more apt to say it's better off to go to the hospital and have everything be fine."

PCP "The other thing is, I think the nurses are trained to be more conservative than physicians in their advice and their guidance, so there might have been a lot more advice given at that home visit about, you know, like if any of these things happen, go to the emergency department right away, whereas the primary care follow-up, they might have gotten a little bit more nuanced advice from the pediatrician or just ... more like, no, it's fine, call us if you have questions."

"I think it depends on what order they hear stuff in. And then ... no matter what you say sometimes, they hear only the beginning or only the end or whatever ... so they didn't hear, 'Call PCP or go to ER,' they just heard, 'Go to ER.'."

Abbreviations: ED, emergency department; ER, emergency room; HM, hospital medicine physicians; PCP, primary care physicians; RN, home care pediatric certified registered nurses.

Theme 3: Providing Context for and Framing of Red Flags Physicians and nurses suggested providing more context to "red flag" instructions and education. RNs emphasized that some families seemed to benefit from the opportunity to discuss their postdischarge concerns with a medical professional. Others appreciated concrete written instructions that spelled out how to respond in certain situations (Table 3).

\section{Minor Themes}

Three minor themes were revealed regarding intervention design improvement (Table 1): (1) streamlining the discharge process; (2) improving the definition of the scope and goal of intervention; and (3) extending inpatient team expertise post discharge. Supportive quotations can be found in Appendix 3.

\section{DISCUSSION}

When stakeholders were asked about why postdischarge RN visits led to increased postdischarge urgent healthcare visits, they questioned the appropriateness of the reutilization events, wondered about the lack of context for the warning signs that nurses provided families as part of the intervention, worried that families were encouraged to return to the hospital because of the ties of the trial to the hospital, and suggested that RNs had a low threshold to refer patients back to the hospital. When asked about how to design an improved nurse visit to better support families, stakeholders emphasized improving communication, individualizing the visit, and providing context around the red-flag discussion, enabling more nuanced instructions about how to respond to specific events.

A synthesis of themes suggests that potential drivers for increased utilization rates may lie in the design and goals of the initial project. The intervention was designed to support families and enhance education after discharge, with components derived from pretrial focus groups with families after a hospital discharge. ${ }^{8}$ The intervention was not designed to divert patients from the ED nor did it enhance access to the PCP. A second trial of the intervention adapted to a phone call also failed to decrease reutilization rates. ${ }^{9}$ Both physician stakeholder groups perceived that the intervention directed traffic back to the hospital because of the intervention design. Coupled with the perception that the red flags may have changed a family's threshold for seeking care and/or that an RN may be more 
TABLE 3. Suggestions for Improving the Intervention: Major Themes and Illustrative Quotations

\begin{tabular}{|c|c|c|}
\hline Theme & Stakeholder Type* & Illustrative Quotation \\
\hline \multirow[t]{3}{*}{$\begin{array}{l}\text { Need for improved postdischarge } \\
\text { communication }\end{array}$} & Parent & $\begin{array}{l}\text { "[Multiple phone calls were] our biggest issue. [We were] just trying to communicate, [and] I thought there was a barrier there. } \\
\text {... The physician didn't want to speak with [us]." }\end{array}$ \\
\hline & $\mathrm{HM}$ & $\begin{array}{l}\text { "But I think it gets back to ... with whom should that nurse communicate? And I think that's, that gets tricky, because sometime } \\
\text { the inpatient physician is the best person, but then there are probably a lot of times where the primary care doctor would really } \\
\text { benefit from being in the know as well.... I don't think that was often part of what would happen at the nurse visits, so ..." }\end{array}$ \\
\hline & & $\begin{array}{l}\text { "I had a couple of cases where they've called me [during the visit] just because there's been a lot of discrepancies to what the } \\
\text { pediatrician doesn't know or the fact that they can't get ahold of the appropriate pediatrician to get those orders. And I am } \\
\text { more than willing to help.... I believe there are certain difficulties because I can't say, 'Well, you need to do this right or enforce } \\
\text { something because I'm not their primary caregiver.' " }\end{array}$ \\
\hline
\end{tabular}

"I think we probably have some idea of what primary care docs would want to know about or want to be notified about, and... there are some things that are just, our job as the inpatient providers, and that it's our responsibility to clean up any issues that result from inpatient stay."

PCP "So I think if the nurse could say, 'Well, I talked to your PCP and they want you to call them if you see these warning signs' or 'Your PCP doctor got the discharge summary, and they want you to call if you see these warning signs,', and you might reinforce because if it comes across as a personal thing, then they feel like, 'Oh, the doctor knows about me.' They want to know that.... Oh, well, the hospital knows about me, the hospital wants to see me back."

"The home nurse could even call the primary care nurse from the home ... and like sort of summarize the situation and, you know, even let the parent talk to the nurse so that the nurse could say to the parent, 'You know, they're doing better, and certainly don't hesitate to call me if you have any worries.'"

RN "One of the things ... is that not every primary care is tied into our system. So they get no idea what happened at our visit."

"Well, I think if there would have been some type of ... discharge plan or whatever on the floor, I get they're busy. I understand that. But even if they could have made a phone call to the primary care team physician and said this first one is admitted for this, then they'll get a follow-up for this, and this is what you can expect."

Individualizing home visits—one size does not Parent fit all

"Again, it [need for visit] just depends on the circumstance. I mean, it would, you know, I'm not opposed to it."

"I think anytime a kid is discharged after having major surgery, especially like my daughter had brain surgery, so I think that you're always a little nervous and a little stressed. And, I mean, it's traumatizing, especially if you're worried about your kid."

HM "There [are] some diagnoses and family scenarios where I was almost sure that that would be a valuable intervention."

"Like one of those families that just makes you nervous and that, you know, needs a lot of reassurance or needs a lot of like reinforcement of education."

PCP "I mean, I think you would want to decide what kind, what visits are going to be valuable and what are not, you know."

"Yeah, I think it's, you know, yeah, you could say all asthmatics. That's easier, all new onset diabetics, or all mental health discharges. But then the routine stuff, not all bronchiolitis would, I would think needs it, but, yeah, like a 6-week-old expreemie, yeah, right? So I think it's situational to none. I don't know that I would just blanket universal."

"I could see it being valuable for like a new onset diabetic or a patient new with epilepsy, like teaching the family what to do if they have a seizure, safety measures around the house."

RN $\quad$ RN 1: "Toddlers and infants are very beneficial because these parents are always questioning their own judgment about what to do because they're very frightened."

RN 2: "I think the respiratory stuff too. "

RN 1: "Because you just can't stand to hear your child sound like that and you need to breathe to live. So they get, I think they're really nervous about that."

RN: "Like, I guess, we know, we assess them a little further away from their discharge."

RN: "I mean, I think sometimes ... will reassure them, and if they felt maybe he was okay or my child is okay if you get discharged, but maybe 3, 4, 5 [days], a week later, and maybe they're rethinking, I don't know."

Continued on page 524

apt to refer back to care, this failure to push utilization to the primary care office may explain the unexpected trial results. Despite the stakeholders' perception of enhanced connection back to the hospital as a result of the nurse visit, in analysis of visit referral patterns, a referral was made directly back to the ED in only 4 of the 651 trial visits (Tubbs-Cooley H, Riddle SR,
Gold JM, et al.; under review. Pediatric clinical and social concerns identified by home visit nurses in the immediate postdischarge period 2020).

Both $\mathrm{H} 2 \mathrm{O}$ trials demonstrated improved recall of red flags by parents who received the intervention, which may be important given the stakeholders' perspectives that the red flags 
TABLE 3. Suggestions for Improving the Intervention: Major Themes and Illustrative Quotations (continued)

\begin{tabular}{lll}
\hline Theme & Stakeholder Type* & Illustrative Quotation \\
\hline Providing context for and framing of red flags & PCP & $\begin{array}{l}\text { "So it, I mean, because like the Barton Schmitt Nursing Guidelines, Triage Guidelines, have like good, like call } 911, \text { go to the } \\
\text { emergency room now, be seen in the office today, be seen in the office tomorrow, today or tomorrow, eventually.... So that's } \\
\text { kind of my mental framework for that." }\end{array}$ \\
\hline
\end{tabular}

"So if you can, the nurse [should be] almost from the primary care office rather than from the hospital. And then the instructions are very much tailored to, 'If you see this and this, you know, call your doctor' ... and the emergency room being sort of that last tier."

"When you give red flags, or you call me if this or this, but it lacks context, like, okay, their breathing went to 70, but their fever was 102. What is it when the fever is better? Oh, the breathing is back down. Okay. That's okay, stay home, versus no fever, and the breathing is 70 . You know, so I think everything has a context, and details that might be hard to really teach."

RN

\begin{abstract}
"There were times where ... we would give the parents a lot of education about using the red flags and ... I said, 'You're not red enough for me to call the doctor, but you're in a yellow zone. You are not green for go, you are in the yellow zone. You need to have caution. You need to watch him.' And that's what I would use with talking with them. And I said, 'You need to refer to these [red flags]."
\end{abstract}

RN 1: "Is our strategy she could have had the family work through it before they got to the point where they needed to call somebody? So try $X, Y$, and $Z$ if they're not doing this, and then call your doctor, would that like add an extra layer to your red flags?"

RN 2: "And I don't know ... I mean, some moms can't, you know, they can't figure that out at times. You know, we just got to [say], 'It's either this or this.'"

Abbreviations: HM, hospital medicine physicians; PCP, Primary care physicians; RN, home care pediatric certified registered nurses.

may not have been contextualized well enough. Yet neither trial demonstrated any differences in postdischarge coping or time to return to normal routine. In interviews with parents, despite the clearly stated results of increased reutilization, intervention parents endorsed a desire for a home visit in the future, raising the possibility that our outcome measures did not capture parents' priorities adequately.

When asked to recommend design improvements of the intervention, 2 major themes (improvement in communication and individualization of visits) were discussed by all stakeholder groups, providing actionable information to modify or create new interventions. Focus groups with clinicians suggested that communication challenges may have influenced reutilization likelihood during the postdischarge period. RNs expressed uncertainty about whom to call with problems or questions at the time of a home visit. This was compounded by difficulty reaching physicians. Both hospital medicine physicians and PCPs identified system challenges including questions of patient ownership, variable PCP practice communication preferences, and difficulty in identifying a partnered staff member (on either end of the inpatient-outpatient continuum) who was familiar with a specific patient. While the communication issues raised may reflect difficulties in our local healthcare system, there is broad evidence of postdischarge communication challenges. In adults, postdischarge communication failures between home health staff and physicians are associated with an increased risk of readmission. ${ }^{10}$ The real or perceived lack of communication between inpatient and outpatient providers can add to parental confusion post discharge. ${ }^{11}$ Although there have been efforts to improve the reliability of communication across this gulf, 12,13 it is not clear whether changes to discharge communication could help to avoid pediatric reutilization events. ${ }^{14}$
The theme of individualization of the home nurse visit is consistent with evidence regarding the impact of focusing the intervention on patients with specific diagnoses or demographics. In adults, reduced reutilization associated with postdischarge home nurse visits has been described in specific populations such as patients with heart failure and chronic obstructive pulmonary disease..$^{15}$ Impact of home nurse visits on patients within diagnosis-specific populations with certain demographics (such as advanced age) has also been described. ${ }^{16}$ In the pediatric population, readmission rates vary widely by diagnosis. ${ }^{17}$ A systematic review of interventions to reduce pediatric readmissions found increased impact of discharge interventions in specific populations (asthma, oncology, and neonatal intensive care patients). ${ }^{3}$

Next steps may lie in interventions in targeted populations that function as part of a care continuum bridging the patient from the inpatient to the outpatient setting. A home nurse visit as part of this discharge structure may prove to have more impact on reducing reutilization. One population that accounts for a large proportion of readmissions and in which there has been recent focus on discharge transition of care has been children with medical complexity. ${ }^{18}$ This group was largely excluded from the $\mathrm{H} 2 \mathrm{O}$ trial. Postdischarge home nurse visits in this population have been found to be feasible and address many questions and problems, but the effect on readmission is less clear. ${ }^{19}$ Family priorities and preferences related to preparation for discharge, including family engagement, respect for discharge readiness, and goal of returning to normal routines, may be areas on which to focus with future interventions in this population. ${ }^{20}$ In summary, although widespread postdischarge interventions (home nurse visit ${ }^{4}$ and nurse telephone call') have not been found to be effective, targeting interventions to specific populations by diagnosis or demographic factors may 
prove to be more effective in reducing pediatric reutilization.

There were several strengths to this study. This qualitative approach allowed us to elucidate potential explanations for the $\mathrm{H} 2 \mathrm{O}$ trial results from multiple perspectives. The multidisciplinary composition of our analytic team and the use of an iterative process sparked diverse contributions in a dynamic, ongoing discussion and interpretation of our data.

This study should be considered in the context of several limitations. For families and RNs, there was a time lag between participation in the trial and participation in the qualitative study call or focus group that could lead to difficulty recalling details. Only families who received the intervention could give opinions on their experience of the nurse visit, while families in the control group were asked to hypothesize. Focus groups with hospital medicine physicians and PCPs were purposive samples, and complete demographic information of participants was not collected.

\section{CONCLUSION}

Key stakeholders reflecting on a postdischarge $\mathrm{RN}$ visit trial suggested multiple potential explanations for the unexpected increase in reutilization in children randomized to the intervention. Certain participants questioned whether all reutilization events were appropriate or necessary. Others expressed concerns that the $\mathrm{H} 2 \mathrm{O}$ intervention lacked context and directed children back to the hospital instead of the PCP. Parents, PCPs, hospital medicine physicians, and RNs all suggested that future transition-focused interventions should enhance post-

\section{References}

1. Auger KA, Simon TD, Cooperberg D, et al. Summary of STARNet: seamless transitions and (re)admissions network. Pediatrics. 2015;135(1):164-175. https://doi.org/10.1542/peds.2014-1887

2. Toomey SL, Peltz A, Loren S, et al. Potentially preventable 30-day hospital readmissions at a Children's Hospital. Pediatrics. 2016;138(2). https://doi. org/10.1542/peds.2015-4182

3. Auger KA, Kenyon CC, Feudtner C, Davis MM. Pediatric hospital discharge interventions to reduce subsequent utilization: a systematic review. J Hosp Med. 2014;9(4):251-260. https://doi.org/10.1002/jhm.2134

4. Auger KA, Simmons JM, Tubbs-Cooley HL, et al. Postdischarge nurse home visits and reuse: the Hospital to Home Outcomes (H2O) trial. Pediatrics. 2018;142(1). https://doi.org/10.1542/peds.2017-3919

5. Tubbs-Cooley HL, Pickler RH, Simmons JM, et al. Testing a post-discharge nurse-led transitional home visit in acute care pediatrics: the Hospital-To-Home Outcomes (H2O) study protocol. J Adv Nurs. 2016;72(4):915-925. https://doi.org/10.1111/jan.12882

6. Guest G. Collecting Qualitative Data: A Field Manual for Applied Research. SAGE Publications, Inc.; 2013.

7. Patton M. Qualitative Research and Evaluation Methods. 4th ed. SAGE Publications, Inc.; 2014.

8. Solan $L G$, Beck AF, Brunswick $S A$, et al. The family perspective on Hospital to Home Transitions: a qualitative study. Pediatrics. 2015;136(6):e1539-e1549. https://doi.org/10.1542/peds.2015-2098

9. Auger KA, Shah SS, Tubbs-Cooley HL, et al. Effects of a 1-time nurse-led telephone call after pediatric discharge: the $\mathrm{H} 2 \mathrm{O} \| \mathrm{I}$ randomized clinical trial. JAMA Pediatr. 2018;172(9):e181482. https://doi.org/10.1001/jamapediatrics.2018.1482

10. Pesko MF, Gerber LM, Peng TR, Press MJ. Home health care: nurse-physician communication, patient severity, and hospital readmission. Health Serv Res. 2018;53(2):1008-1024. https://doi.org/10.1111/1475-6773.12667

11. Solan LG, Beck AF, Shardo SA, et al. Caregiver perspectives on communication during hospitalization at an academic pediatric institution: a qualitative discharge communication, strengthen connection to the PCP, and be more effectively tailored to the needs of the individual patient and family.

\section{Acknowledgments}

Collaborators: H2O Trial Study Group: Joanne Bachus, BSN, RN, Department of Patient Services, Cincinnati Children's Hospital Medical Center, Cincinnati, Ohio; Monica L Borell, BSN, RN, Department of Patient Services, Cincinnati Children's Hospital Medical Center, Cincinnati, Ohio; Lenisa V Chang, MA, PhD; Patricia Crawford, RN, Department of Patient Services, Cincinnati Children's Hospital Medical Center, Cincinnati, Ohio; Sarah A Ferris, BA, Division of Hospital Medicine, Cincinnati Children's Hospital Medical Center, Cincinnati, Ohio; Judy A Heilman BSN, RN, Department of Patient Services, Cincinnati Children's Hospital Medical Center, Cincinnati, Ohio; Jane C Khoury, PhD, Division of Biostatistics and Epidemiology, Cincinnati Children's Hospital Medical Center, Cincinnati, Ohio; Karen Lawley, BSN, RN, Department of Patient Services, Cincinnati Children's Hospital Medical Center, Cincinnati, Ohio; Lynne O’Donnell, BSN, RN, Department of Patient Services, Cincinnati Children's Hospital Medical Center, Cincinnati, Ohio; Hadley S Sauers-Ford, MPH, Department of Pediatrics, UC Davis Health, Sacramento, California; Anita N Shah, DO, MPH, Division of Hospital Medicine, Cincinnati Children's Hospital Medical Center, Cincinnati, Ohio; Lauren G Solan, MD, Med, University of Rochester, Rochester, New York; Heidi J Sucharew, PhD, Division of Biostatistics and Epidemiology, Cincinnati Children's Hospital Medical Center, Cincinnati, Ohio; Karen P Sullivan, BSN, RN, Department of Patient Services, Cincinnati Children's Hospital Medical Center, Cincinnati, Ohio; Christine M White, MD, MAT, Division of Hospital Medicine, Cincinnati Children's Hospital Medical Center, Cincinnati, Ohio

Disclosure: The authors have no potential conflicts of interest relevant to this article to disclose.

Funding: This work was supported through a Patient-Centered Outcomes Research Institute (PCORI) Award (HIS-1306-00811).

study. J Hosp Med. 2018;13(5):304-311. https://doi.org/10.12788/jhm.2919

12. Zackoff MW, Graham C, Warrick D, et al. Increasing PCP and hospital medicine physician verbal communication during hospital admissions. Hosp Pediatr. 2018;8(4):220-226. https://doi.org/10.1542/hpeds.2017-0119

13. Mussman GM, Vossmeyer MT, Brady PW, et al. Improving the reliability of verbal communication between primary care physicians and pediatric hospitalists at hospital discharge. J Hosp Med. 2015;10(9):574-580. https://doi. org/10.1002/jhm.2392

14. Coller RJ, Klitzner TS, Saenz AA, et al. Discharge handoff communication and pediatric readmissions. J Hosp Med. 2017;12(1):29-35. https://doi. org/10.1002/jhm.2670

15. Yang F, Xiong ZF, Yang $C$, et al. Continuity of care to prevent readmissions for patients with chronic obstructive pulmonary disease: a systematic review and meta-analysis. COPD. 2017;14(2):251-261. https://doi.org/10.1080/15412555.2 016.1256384

16. Finlayson K, Chang AM, Courtney MD, et al. Transitional care interventions reduce unplanned hospital readmissions in high-risk older adults. BMC Health Serv Res. 2018;18(1):956. https://doi.org/10.1186/s12913-018-3771-9

17. Berry JG, Toomey SL, Zaslavsky AM, et al. Pediatric readmission prevalence and variability across hospitals. JAMA. 2013;309(4):372-380. https://doi. org/10.1001/jama.2012.188351

18. Coller RJ, Nelson BB, Sklansky DJ, et al. Preventing hospitalizations in children with medical complexity: a systematic review. Pediatrics. 2014;134(6):e1628-e1647. https://doi.org/10.1542/peds.2014-1956

19. Wells S, O'Neill M, Rogers J, et al. Nursing-led home visits post-hospitalization for children with medical complexity. J Pediatr Nurs. 2017;34:10-16. https://doi.org/10.1016/j.pedn.2017.03.003

20. Leyenaar JK, O'Brien ER, Leslie LK, Lindenauer PK, Mangione-Smith RM. Families' priorities regarding hospital-to-home transitions for children with medical complexity. Pediatrics. 2017;139(1). https://doi.org/10.1542/ peds.2016-1581 